\title{
The H-index and First-author H-index of Chinese Scholars in LIS
}

\author{
Yuecong Chen ${ }^{1}$, Zongqi Liu ${ }^{2}$ \\ 1,2 Library of North China Electric Power University, Beijing 102206 \\ 1'cyc-79@163.com1, 2lzq@ncepu.edu.cn2
}

Keywords: H-index; Library and Information Science (LIS); JCR

\begin{abstract}
Based on the journals of JCR Social Sciences Edition--INFORMATION SCIENCE \& LIBRARY SCIENCE category from 2003 to 2014, the Chinese authors' 2793 articles in Library and Information Science (LIS) were collected and analyzed on Web of Science (WOS). First, it ranked the first Chinese authors, and selected 76 authors with more than 5 articles as sample authors. Then it collected the sample authors' articles on WOS, and the h-index and first author h-index were listed and analyzed.
\end{abstract}

\section{Introduction}

The h-index is an author-level metric that attempts to measure both the productivity and citation impact of the publications of a scientist or scholar. The $\mathrm{h}$-index of an individual author is defined as the maximum number of papers $h$ by a scientist where each of those papers has received $h$ or more citations. The index was suggested in 2005 by Jorge E. Hirsch, a physicist at UCSD, as a tool for determining theoretical physicists' relative quality and is sometimes called the Hirsch index or Hirsch number.

It is recognized that the total number of publications does not provide a measure of the quality of these publications, while the total number of citations may be biased by relatively few, very highly cited works. The h-index is intended to measure simultaneously the quality and quantity of scientific output. The advantages of the h-index include that it is mathematically simple, it may be applied to any level of aggregation, it is a robust indicator, and it improves the quality of published work. Some criticisms of the h-index have included that it is bounded by the total number of publications, it is limited by the completeness of the database used to count, and it does not take into account the number of coauthors of each paper. This paper analyzed the Chinese authors' h-index in LIS based on JCR journals from 2003 to 2014.

Web of Science (previously known as (ISI) Web of Knowledge, also as WOS) is an online subscription-based scientific citation indexing service maintained by Thomson Reuters that provides a comprehensive citation search. It consists of SCI, SSCI, A\&HCI, CPCI-S, CPCI-SSH, and so on a series of Citation Database. It includes more than 11000 kinds of the most influential and high quality journals in the world.

Journal Citation Reports (JCR) is an annual publication by ISI in two editions. JCR Science Edition contains data about more than 8,000 journals in science and technology. JCR Social Sciences Edition contains data about more than 2,600 journals in the social sciences.

The articles used in this paper came from the JCR Social Sciences Edition (2003-2014), and it analyzed the high produced Chinese authors' h-index and the first-author h-index of theirs in Library and Information Science (LIS).

\section{Collect the articles from JCR Social Sciences Edition (2003-2014)}

The journals of the category of INFORMATION SCIENCE \& LIBRARY SCIENCE in JCR Social Sciences Edition were collected annually from 2003 to 2014 . All the articles in the above journals were searched in Web of Science, and the region was limited to PEOPLES R CHINA (China mainland, Hong Kong and TAIWAN). 
Table 1 The articles of Chinese authors from 2003 to 2014

\begin{tabular}{c|c|c|c|c|c|c|c|c|c|c|c|c}
\hline & $\mathbf{2 0 0 3}$ & $\mathbf{2 0 0 4}$ & $\mathbf{2 0 0 5}$ & $\mathbf{2 0 0 6}$ & $\mathbf{2 0 0 7}$ & $\mathbf{2 0 0 8}$ & $\mathbf{2 0 0 9}$ & $\mathbf{2 0 1 0}$ & $\mathbf{2 0 1 1}$ & $\mathbf{2 0 1 2}$ & $\mathbf{2 0 1 3}$ & $\mathbf{2 0 1 4}$ \\
\hline $\begin{array}{c}\text { Number of LIS } \\
\text { journals }\end{array}$ & 55 & 54 & 55 & 53 & 56 & 61 & 66 & 77 & 83 & 85 & 84 & 85 \\
\hline $\begin{array}{c}\text { The articles the } \\
\text { whole world }\end{array}$ & 10286 & 9866 & 9766 & 9534 & 9446 & 9255 & 8060 & 9205 & 9475 & 8808 & 9450 & 9670 \\
\hline Chinese articles & 81 & 75 & 99 & 124 & 134 & 191 & 234 & 244 & 318 & 386 & 447 & 460 \\
\hline
\end{tabular}

Form Table 1, there were 112821 articles all over the world, and the number of Chinese authors was no more than 100 in 2003, and increased to more than 400 in 2014. There were totally 2793 articles of Chinese authors in 12 years from 2003 to 2014, which were the sample articles of this paper.

\section{Rank the High Produced Chinese Authors}

The first Chinese authors were extracted from 2793 sample articles, 1718 different first Chinese authors were counted after merging and duplicate removal. There were 76 authors that with more than 5 articles, and the 76 authors were selected as sample authors of this paper.

Table 2 The 76 sample authors

\begin{tabular}{|c|c|c|c|c|c|c|c|c|}
\hline Ranking & Author & Articles & Ranking & Author & Articles & Ranking & Author & Articles \\
\hline 1 & Huang, Mu-Hsuan & 23 & 27 & Chen, Kuan-nien & 7 & 53 & Zhao, Rongying & 6 \\
\hline 2 & Tsay, Ming-yueh & 17 & 28 & Hung, Shin-Yuan & 7 & 54 & $\begin{array}{c}\text { Cheung, Christy } \\
\text { M. K. }\end{array}$ & 5 \\
\hline 3 & Yu, Guang & 15 & 29 & Li, Jiang & 7 & 55 & $\begin{array}{l}\text { Chuang, } \\
\text { Kun-Yang }\end{array}$ & 5 \\
\hline 4 & Guan, Jiancheng & 14 & 30 & Liu, Rey-Long & 7 & 56 & Fu, Hui-Zhen & 5 \\
\hline 5 & Li, Xia & 13 & 26 & Wu, Ing-Long & 7 & 57 & He, Tianwei & 5 \\
\hline 6 & $\begin{array}{c}\text { Chu, Samuel } \\
\text { Kai-Wah }\end{array}$ & 12 & 26 & Yu, Liangzhi & 7 & 57 & Jin, Yi & 5 \\
\hline 7 & Rousseau, Ronald & 11 & 26 & Zha, Xianjin & 7 & 57 & Lai, Jung-Yu & 5 \\
\hline 7 & Yang, Christopher C. & 11 & 26 & Zhang, Jin & 7 & 57 & Li, Aiguo & 5 \\
\hline 7 & Ye, Fred Y. & 11 & 35 & Chau, Michael & 6 & 57 & Liao, Chechen & 5 \\
\hline 7 & Zhou, Ping & 11 & 35 & Chen, Dar-zen & 6 & 57 & Lin, Tung-Ching & 5 \\
\hline 11 & Davison, Robert M. & 10 & 35 & Chou, Shih-Wei & 6 & 57 & Liu, Duen-Ren & 5 \\
\hline 11 & $\mathrm{Hu}$, Xiaojun & 10 & 35 & Chou, Tzu-Chuan & 6 & 57 & Liu, Xuan Zhen & 5 \\
\hline 11 & Liang, Liming & 10 & 35 & Gao, Xia & 6 & 57 & Ma, Feicheng & 5 \\
\hline 11 & Lin, Hsiu-Fen & 10 & 35 & Ho, Yuh-Shan & 6 & 57 & Pei, Tao & 5 \\
\hline 11 & Liu, Yuxian & 10 & 35 & Hong, Weiyin & 6 & 57 & Ting, I-Hsien & 5 \\
\hline 11 & Wang, Xianwen & 10 & 35 & Kao, Chiang & 6 & 57 & $\begin{array}{c}\text { Tseng, } \\
\text { Yuen-Hsien }\end{array}$ & 5 \\
\hline 17 & Chen, Yen-Liang & 9 & 35 & Leydesdorff, Loet & 6 & 57 & Wang, Weiquan & 5 \\
\hline 17 & Chen, Yu-Shan & 9 & 35 & Liu, Xiaoping & 6 & 57 & Wang, Yuandi & 5 \\
\hline 17 & Chiu, Chao-Min & 9 & 35 & $\begin{array}{l}\text { Lowry, Paul } \\
\text { Benjamin }\end{array}$ & 6 & 57 & Wei, Chih-Ping & 5 \\
\hline 17 & Wang, Eric T. G. & 9 & 35 & Qiu, Junping & 6 & 57 & Wu, Dan & 5 \\
\hline 17 & Wang, Yi-Shun & 9 & 35 & Tsai, Chih-Fong & 6 & 57 & Wu, I-Chin & 5 \\
\hline 17 & Zhang, Lin & 9 & 35 & Vaughan, Liwen & 6 & 57 & Xu, Yan & 5 \\
\hline 23 & Chen, Chuanfu & 8 & 35 & Wu, Jen-Her & 6 & 57 & Zhao, Star X. & 5 \\
\hline 23 & Xia, Jun & 8 & 35 & Wu, Ming-der & 6 & 57 & Zhou, Tao & 5 \\
\hline 23 & Yu, Shien-Chiang & 8 & 35 & Yan, Yalan & 6 & & & \\
\hline 26 & Chang, Hsin Hsin & 7 & 35 & Yang, Chyan & 6 & & & \\
\hline
\end{tabular}




\section{The Analysis of Sample Authors' Papers and H-Index in Wos}

The papers of 76 sample authors in WOS were collected and the h-index of each author was counted in WOS, too.

Table 3 The articles and h-index of 76 sample authors

\begin{tabular}{|c|c|c|c|c|c|c|c|c|}
\hline Author & h-index & Articles & Author & h-index & Articles & Author & h-index & Articles \\
\hline Leydesdorff, Loet & 32 & 197 & Chen, Dar-zen & 10 & 89 & Xu, Yan & 5 & 24 \\
\hline Ho, Yuh-Shan & 30 & 106 & Vaughan, Liwen & 10 & 32 & Xia, Jun & 4 & 8 \\
\hline Guan, Jiancheng & 18 & 76 & Davison, Robert M. & 9 & 33 & Zhang, Jin & 4 & 16 \\
\hline Li, Xia & 16 & 72 & Chau, Michael & 9 & 40 & Chou, Tzu-Chuan & 4 & 21 \\
\hline Rousseau, Ronald & 16 & 135 & Chou, Shih-Wei & 9 & 19 & Yan, Yalan & 4 & 20 \\
\hline $\begin{array}{c}\text { Yang, Christopher } \\
\text { C. } \\
\end{array}$ & 16 & 116 & Hong, Weiyin & 9 & 13 & Wang, Yuandi & 4 & 25 \\
\hline Lin, Hsiu-Fen & 16 & 29 & Liao, Chechen & 9 & 27 & Li, Jiang & 3 & 14 \\
\hline Chen, Yen-Liang & 16 & 76 & Wei, Chih-Ping & 9 & 43 & Liu, Rey-Long & 3 & 20 \\
\hline $\begin{array}{c}\text { Cheung, Christy M. } \\
\text { K. }\end{array}$ & 16 & 55 & Zhou, Ping & 8 & 16 & Yu, Liangzhi & 3 & 9 \\
\hline Liu, Duen-Ren & 16 & 81 & Yu, Guang & 7 & 31 & Zha, Xianjin & 3 & 29 \\
\hline Chiu, Chao-Min & 15 & 26 & Liang, Liming & 7 & 22 & Wu, Ming-der & 3 & 7 \\
\hline Wang, Eric T. G. & 14 & 35 & Fu, Hui-Zhen & 7 & 17 & He, Tianwei & 3 & 7 \\
\hline Wang, Yi-Shun & 14 & 34 & Lai, Jung-Yu & 7 & 19 & Li, Aiguo & 3 & 5 \\
\hline Chang, Hsin Hsin & 14 & 38 & Lin, Tung-Ching & 7 & 23 & Ma, Feicheng & 3 & 32 \\
\hline Kao, Chiang & 14 & 49 & Pei, Tao & 7 & 38 & Wu, Dan & 3 & 22 \\
\hline Tsai, Chih-Fong & 14 & 65 & Ye, Fred Y. & 6 & 38 & Wu, I-Chin & 3 & 12 \\
\hline Chen, Yu-Shan & 13 & 51 & $\mathrm{Hu}$, Xiaojun & 6 & 32 & Zhao, Star X. & 3 & 14 \\
\hline Liu, Xiaoping & 13 & 52 & Liu, Yuxian & 6 & 18 & Chen, Chuanfu & 2 & 15 \\
\hline Wu, Jen-Her & 13 & 31 & Wang, Xianwen & 6 & 21 & Chen, Kuan-nien & 2 & 10 \\
\hline Zhou, Tao & 13 & 43 & Gao, Xia & 6 & 10 & Jin, Yi & 2 & 5 \\
\hline Hung, Shin-Yuan & 12 & 37 & Chuang, Kun-Yang & 6 & 15 & Liu, Xuan Zhen & 2 & 6 \\
\hline $\begin{array}{c}\text { Lowry, Paul } \\
\text { Benjamin }\end{array}$ & 12 & 44 & Tseng, Yuen-Hsien & 6 & 20 & Yu, Shien-Chiang & 1 & 5 \\
\hline Huang, Mu-Hsuan & 11 & 75 & Tsay, Ming-yueh & 5 & 22 & Zhao, Rongying & 1 & 14 \\
\hline Yang, Chyan & 11 & 60 & $\begin{array}{c}\text { Chu, Samuel } \\
\text { Kai-Wah }\end{array}$ & 5 & 20 & Ting, I-Hsien & 1 & 19 \\
\hline Zhang, Lin & 10 & 47 & Qiu, Junping & 5 & 31 & & & \\
\hline Wu, Ing-Long & 10 & 27 & Wang, Weiquan & 5 & 8 & & & \\
\hline
\end{tabular}

Leydesdorff, Loet; Ho, Yuh-Shan and Guan, Jiancheng had the highest h-index 32, 30 and 18 respectively. We could conclude that the h-indexs of Chinese LIS authors were not very high, only 28 authors' h-index were more than 10 .

\section{The Analysis of Sample Authors' First Author H-Index in WOS}

The first authors' articles were collected among the above articles for each sample author. And the h-index was recounted according to the first authors' articles, which we called it first-author h-index or h'-index. 
Table 4 The h-index and h'-index of 76 sample authors

\begin{tabular}{|c|c|c|c|c|c|c|c|c|}
\hline Author & h-index & $h^{\prime}$-index & Author & h-index & $h^{\prime}$-index & Author & h-index & $h^{\prime}$-index \\
\hline Leydesdorff, Loet & 32 & 27 & Chen, Dar-zen & 10 & 4 & Xu, Yan & 5 & 5 \\
\hline Ho, Yuh-Shan & 30 & 21 & Vaughan, Liwen & 10 & 8 & Xia, Jun & 4 & 4 \\
\hline Guan, Jiancheng & 18 & 15 & Davison, Robert M. & 9 & 5 & Zhang, Jin & 4 & 4 \\
\hline Li, Xia & 16 & 10 & Chau, Michael & 9 & 7 & Chou, Tzu-Chuan & 4 & 3 \\
\hline Rousseau, Ronald & 16 & 7 & Chou, Shih-Wei & 9 & 9 & Yan, Yalan & 4 & 3 \\
\hline $\begin{array}{l}\text { Yang, Christopher } \\
\text { C. }\end{array}$ & 16 & 11 & Hong, Weiyin & 9 & 7 & Wang, Yuandi & 4 & 3 \\
\hline Lin, Hsiu-Fen & 16 & 16 & Liao, Chechen & 9 & 7 & Li, Jiang & 3 & 3 \\
\hline Chen, Yen-Liang & 16 & 14 & Wei, Chih-Ping & 9 & 7 & Liu, Rey-Long & 3 & 3 \\
\hline $\begin{array}{c}\text { Cheung, Christy M. } \\
\text { K. }\end{array}$ & 16 & 8 & Zhou, Ping & 8 & 6 & Yu, Liangzhi & 3 & 3 \\
\hline Liu, Duen-Ren & 16 & 12 & Yu, Guang & 7 & 4 & Zha, Xianjin & 3 & 3 \\
\hline Chiu, Chao-Min & 15 & 11 & Liang, Liming & 7 & 5 & Wu, Ming-der & 3 & 3 \\
\hline Wang, Eric T. G. & 14 & 12 & Fu, Hui-Zhen & 7 & 5 & He, Tianwei & 3 & 2 \\
\hline Wang, Yi-Shun & 14 & 10 & Lai, Jung-Yu & 7 & 7 & Li, Aiguo & 3 & 3 \\
\hline Chang, Hsin Hsin & 14 & 14 & Lin, Tung-Ching & 7 & 6 & Ma, Feicheng & 3 & 2 \\
\hline Kao, Chiang & 14 & 14 & Pei, Tao & 7 & 4 & Wu, Dan & 3 & 1 \\
\hline Tsai, Chih-Fong & 14 & 13 & Ye, Fred Y. & 6 & 5 & Wu, I-Chin & 3 & 2 \\
\hline Chen, Yu-Shan & 13 & 12 & $\mathrm{Hu}$, Xiaojun & 6 & 5 & Zhao, Star X. & 3 & 3 \\
\hline Liu, Xiaoping & 13 & 8 & Liu, Yuxian & 6 & 5 & Chen, Chuanfu & 2 & 2 \\
\hline Wu, Jen-Her & 13 & 12 & Wang, Xianwen & 6 & 5 & Chen, Kuan-nien & 2 & 2 \\
\hline Zhou, Tao & 13 & 12 & Gao, Xia & 6 & 4 & Jin, Yi & 2 & 2 \\
\hline Hung, Shin-Yuan & 12 & 10 & Chuang, Kun-Yang & 6 & 4 & Liu, Xuan Zhen & 2 & 2 \\
\hline $\begin{array}{l}\text { Lowry, Paul } \\
\text { Benjamin }\end{array}$ & 12 & 8 & Tseng, Yuen-Hsien & 6 & 6 & Yu, Shien-Chiang & 1 & 1 \\
\hline Huang, Mu-Hsuan & 11 & 7 & Tsay, Ming-yueh & 5 & 5 & Zhao, Rongying & 1 & 1 \\
\hline Yang, Chyan & 11 & 7 & $\begin{array}{l}\text { Chu, Samuel } \\
\text { Kai-Wah }\end{array}$ & 5 & 5 & Ting, I-Hsien & 1 & 0 \\
\hline Zhang, Lin & 10 & 6 & Qiu, Junping & 5 & 2 & & & \\
\hline Wu, Ing-Long & 10 & 10 & Wang, Weiquan & 5 & 3 & & & \\
\hline
\end{tabular}

The first author h-index more than 10 was listed in Table 5 with 19 authors. The articles of Lin, Hsiu-Fen; Chang, Hsin Hsin; Kao, Chiang and Wu, Ing-Long were all first author articles, so the $\mathrm{h}$ '-indexes were not decreased in these four authors. But the difference of articles and first author articles of Ho, Yuh-Shan; Yang, Christopher $\mathrm{C}$ and Li, Xia were more than the others, so the h'-indexes decreased significantly, about 30\%. The h'-index of other 12 authors also decreased in varying degrees. 
Table 5 The comparison of first and not first author h-index

\begin{tabular}{c|c|c|c|c|c}
\hline Author & Articles & Articles' & h-index & $\mathbf{h}^{\prime}$-index & h-index decrease in $\%$ \\
\hline Leydesdorff, Loet & 197 & 105 & 32 & 27 & $-15.63 \%$ \\
\hline Ho, Yuh-Shan & 106 & 64 & 30 & 21 & $-30.00 \%$ \\
\hline Lin, Hsiu-Fen & 29 & 28 & 16 & 16 & $0.00 \%$ \\
\hline Guan, Jiancheng & 76 & 40 & 18 & 15 & $-16.67 \%$ \\
\hline Chen, Yen-Liang & 76 & 47 & 16 & 14 & $-12.50 \%$ \\
\hline Chang, Hsin Hsin & 38 & 37 & 14 & 14 & $0.00 \%$ \\
\hline Kao, Chiang & 49 & 43 & 14 & 14 & $0.00 \%$ \\
\hline Tsai, Chih-Fong & 65 & 40 & 14 & 13 & $-7.14 \%$ \\
\hline Chen, Yu-Shan & 51 & 43 & 13 & 12 & $-7.69 \%$ \\
\hline Wang, Eric T. G. & 35 & 19 & 14 & 12 & $-14.29 \%$ \\
\hline Wu, Jen-Her & 31 & 21 & 13 & 12 & $-7.69 \%$ \\
\hline Liu, Duen-Ren & 81 & 41 & 16 & 12 & $-25.00 \%$ \\
\hline Zhou, Tao & 43 & 41 & 13 & 12 & $-7.69 \%$ \\
\hline Yang, Christopher C. & 116 & 53 & 16 & 11 & $-31.25 \%$ \\
\hline Chiu, Chao-Min & 26 & 18 & 15 & 11 & $-26.67 \%$ \\
\hline Li, Xia & 72 & 17 & 16 & 10 & $-37.50 \%$ \\
\hline Wang, Yi-Shun & 34 & 20 & 14 & 10 & $-28.57 \%$ \\
\hline Hung, Shin-Yuan & 37 & 27 & 12 & 10 & 10 \\
\hline Wu, Ing-Long & 27 & 25 & 10 & $10.00 \%$ \\
\hline
\end{tabular}

\section{Conclusion}

The paper collected the Chinese authors' 2793 articles in JCR (2003-2014) of LIS, ranked the first authors of all the articles and selected 76 authors with more than 5 first author articles as sample authors. Then the h-index and h'-index were counted and analyzed on WOS. Most authors h'-index decreased with variety according to the first authors' articles of them.

\section{References}

[1] J. E. Hirsch. An index to quantify an individual's scientific research output that takes into account the effect of multiple coauthorship [J]. Scientometrics, 2010, 85:741-754.

[2] Kelly, C.D.; Jennions, M.D. The h-index and career assessment by numbers [J]. TRENDS in Ecology and Evolution, Vol. 21 No. 4:167-70.

[3] Mousa Yaminfirooz, Hemmat Gholinia. Multiple h-index: a new scientometric indicator [J]. The Electronic Library, 2015, V33, No3: 547 - 556. 